\title{
Defense mechanisms in adolescents at high risk of developing psychosis: an empirical investigation
}

\author{
Tommaso Boldrini, ${ }^{1}$ Gabriele Lo Buglio, ${ }^{1}$ Guido Giovanardi, ${ }^{2,3}$ Vittorio Lingiardi, ${ }^{2}$ Silvia Salcuni ${ }^{1}$ \\ ${ }^{1}$ Department of Developmental and Socialization Psychology, University of Padova, Padova; ${ }^{2}$ Department of Dynamic and Clinical \\ Psychology, Sapienza University of Rome, Rome; ${ }^{3}$ Department of Psychology, University of Campania “Luigi Vanvitelli," Caserta, Italy
}

\begin{abstract}
Research within psychotherapy and psychopathology frameworks has been inspired by the central concept of defense mechanisms, which play a pivotal role in psychoanalysis. Defense specificities have only recently been studied systematically in the context of several clinical diagnoses. The present study aimed to explore the specificities of defense mechanisms in adolescents at ultra-high risk of developing psychosis and test the relationship between defensive functioning and attenuated psychotic symptoms. Twenty-six adolescent inpatients at ultra-high risk of developing psychosis and a matched clinical control group of inpatients not at risk of psychosis were interviewed. Two observer-rater methods, the Defense Mechanism Rating Scales and the Psychotic-Defense Mechanism Rating Scales were applied to interview transcripts to assess the individual use of defenses. Ultra-high-risk patients demonstrated lower defense mechanisms (i.e., total instances of defense mechanisms observed) and lower overall adaptiveness of the defenses presented. When specific differences between groups were observed, psychotic defenses were the sole defenses that could partially discriminate between ultra-high-risk and not at-risk patients. Regarding the relationship between defense mechanisms and subthreshold psychotic symptoms, psychotic defenses were associated with negative and disorganization symptoms, rather than with positive symptoms. The psychological vulnerability of ultra-high-risk patients is discussed and treatment implications for psychotherapy with such challenging patients are addressed.
\end{abstract}

Key words: Defense mechanisms; ultra-high risk for psychosis; at-risk mental state for psychosis; attenuated psychotic syndrome; adolescence.

Correspondence: Tommaso Boldrini, Department of Developmental and Socialization Psychology, University of Padova, via Venezia, 8, 35131, Padova, Italy.

Tel.: +39.0649917674.

E-mail: tommaso.boldrini@unipd.it

Citation: Boldrini, T., Lo Buglio, G., Giovanardi, G., Lingiardi, V., \& Salcuni, S. (2020). Defense mechanisms in adolescents at high risk of developing psychosis: an empirical investigation. Research in Psychotherapy: Psychopathology, Process and Outcome, 23(1), 4-15. doi: $10.4081 /$ ripppo.2020.456

Contributions: TB and SS conceived the research study and analyzed the data. TB wrote the first draft of the manuscript. GLB and SS collected data by coding the interview transcripts. GG contributed to the writing of the manuscript. SS and VL contributed to the interpretation of the results and critically reviewed the final draft of the manuscript.

Conflict of interest: The authors declare that they have no competing interests.

Received for publication: 29 February 2020.

Accepted for publication: 2 April 2020.

This work is licensed under a Creative Commons Attribution NonCommercial 4.0 License (CC BY-NC 4.0).

${ }^{\circ}$ Copyright: the Author(s), 2020

Licensee PAGEPress, Italy

Research in Psychotherapy:

Psychopathology, Process and Outcome 2020; 23:4-15

doi:10.4081/ripppo.2020.456

\section{Introduction}

Defense mechanisms (or 'defenses') are considered clinically valuable objects not only within the psychodynamic tradition, but also in other theoretical orientations (e.g., Di Giuseppe et al., 2019a; Euler et al., 2018; Ziegler, 2016). The current consensus is that defenses are "mechanisms that mediate the individual's reaction to emotional conflicts and to external stressors" (American Psychiatric Association [APA], 2013; p. 819). According to Vaillant (2011), defenses demonstrate some fundamental properties. First, defenses play a central role in mitigating the distressing effects of emotions and mental representations that are generally associated with conflict. Further, they are unconscious, discrete, dynamic, reversible, adaptive, and even creative or pathological. Finally, although the subject is typically unaware, defense mechanisms appear visible, odd, or even annoying to observers. In contrast to coping mechanisms, defense mechanisms operate in a mostly automatic manner, either partially or wholly out of consious awareness. Specifically, subjects can only perceive conscious correlates of defense mechanisms, although expert clinicians may detect and discriminate defense mechanisms. The "capacity for defensive functioning" has recently been included among the 12 categories of basic mental functioning in the second edition of the Psychodynamic Diagnostic Manual (Lingiardi \& McWilliams, 2017). In fact, assessing 
defenses can be particularly useful for both routine clinical work and process-outcome research in psychotherapy (Babl et al., 2019; Di Giuseppe, Perry, Petraglia, Janzen, \& Lingiardi, 2014; Hilsenroth, Katz, \& Tanzilli, 2018; Prout, Malone, Rice, \& Hoffman, 2019). A strong understanding of defenses can assist clinicians in better understanding the strengths and difficulties of patients and helping them make sense of their experiences to develop adaptive responses to internal and external stressors.

Defense mechanisms are categorized and assessed on a continuum from adaptive to pathological. Vaillant (1971), starting from Semrad's (1967) conception of defensive organization, defined defenses with reference to a hierarchical organization, which Perry (1990) later empirically developed by describing seven principal levels of defensive functioning (Table 2). More "mature" or adaptive defenses (e.g., humor, altruism, sublimation, and suppression) usually maximize the awareness of cognitive and affective mental states, as well as of internal and external stressor, allowing an individual to adaptively modulate the expression and gratification of his or her needs and desires. Conversely, less adaptive defenses (e.g., denial, acting out, projective identification, and splitting) may involve conspicuous distortions in the internal representations of self, others, or external reality, and keep potentially threatening ideas, feelings, memories, wishes or fears out of one's awareness (Maffei et al., 1995; Perry, Knoll \& Tran, 2019).

Although the link between defenses and psychopathology is not at all linear (i.e., a defense mechanism can appear in different psychopathological conditions and at very different levels of severity, see Berny et al., 2014), several investigations focused on the relationship between defenses and specific clinical diagnoses, such as bipolar (Kramer, de Roten, Perry, \& Despland, 2009), depressive, and personality disorders (Di Giuseppe et al., 2019b; Perry, Presniak \& Olson, 2013; Presniak, Olson \& MacGregor, 2010), providing relevant insight for psychological treatment for these clinical populations. Conversely, very few empirical studies have investigated defense mechanisms in patients with psychotic disorders (Cichocki, 2008; Januzzi et al., 1997). Their results are contrasting and difficult to generalize due to the clinical heterogeneity of the evaluated patients and the various tools used. The lack of interest in psychotic ${ }^{1}$ defense mechanisms, as well as defense mechanisms in psychotic patients, can be attributed to several factors. First, general interest in

The term "psychotic" as used here, may cause confusion (Lingiardi \& Boldrini, 2019). Depending on the context, it can be understood in a syndromal way, referring to psychiatric diagnosis, or it can refer to the dynamic functioning of the personality, in the sense of Kernberg's (1984) description of the personality organization. In the P-DMRS (see 'Methods' section), psychotic defenses are considered part of the dynamic manifestation of a patient's functioning. defenses has shown "ups and downs" over time (Cramer, 1998; Cramer, 2000), because the concept of defenses - and psychoanalysis more broadly - has been a taboo research paradigm in certain periods of history, and because previous researches has been methodologically flawed. Second, a lack of valid and comprehensive instruments existed to measure psychotic defenses. In this regard, Berny and colleagues (2014) recently proposed a coding system for the evaluation of psychotic defense mechanisms in clinical interview transcripts (Psychotic-Defense Mechanisms Rating Scales [P-DMRS], see "Methods" section) that may overcome limitations of previous assessment methods.

In general, scholars have mostly studied defenses in nonpsychotic samples and more research is needed to establish the role of defense mechanisms in shaping the psychotic experience. In particular, no prior study has investigated the defense specificities of patients At UltraHigh Risk (UHR) of developing psychosis. The prepsychotic or prodromal stage of schizophrenia has been defined as the period of time characterized by increased changes in thinking, feeling, and behaving from the subject's premorbid mental state and functioning up to the time at which psychotic features fist appear (Yung \& McGorry, 1996; Yung et al., 1996). In the last two decades, interest in the early detection of individuals putatively considered at out of consious awareness psychosis has strongly increased. In particular, research has aimed at developing treatments and interventions to improve symptoms and even delay and prevent the onset of a full-fledged disorder (for a review, Andreou, Bailey, \& Borgwardt, 2019; Lieberman, Small, \& Girgis, 2019). Further systematic investigations of UHR subjects' psychological characteristics are critically needed, and the change mechanisms that preventive treatments should address to be effective still need to be fully understood. Given these premises, our general objective was to investigate the defense mechanisms UHR patients use and to obtain useful information for clinicians who provide psychotherapy treatments to youths considered at risk of developing psychosis. Our first hypothesis was that in comparison with non-UHR patients, UHR patients implement more immature defense mechanisms and, in particular, mechanisms that are theoretically involved in the development of the psychotic experience (i.e., psychotic, disavowal, action, and borderline defenses; see Berney, de Roten, Beretta, Kramer, \& Despland, 2014; Costantinides \& Back, 2010). Our second hypothesis was that significant associations exist between defense mechanisms and dimensional markers of the UHR state (i.e., subclinical psychotic symptoms).

\section{Methods}

\section{Participants}

The sample was composed of 26 help-seeking adolescents (nine males, 17 females) recruited in mental illness 
prevention services within mental health departments in Rome and Padova. The criteria for inclusion were as follow: 13 years of age or older, fluent in Italian, IQ > 70, no organic causes for presentation, and no known psychotic episodes. A strictly matched control group was introduced with the matching criteria of gender, age, and years of education, because these variables can influence defensive functioning (Cramer, 2009; Di Giuseppe, Gennaro, Lingiardi, \& Perry, 2019b; Foto-Özdemir, Akdemir \& Çuhadaroglu-Çetin, 2016; Whitty, 2003). Twenty-six non-UHR patients were recruited from the same clinical centers. No significant differences were found in the matching variables between groups (Table 1), either in concomitant clinical diagnoses or in the attachment patterns (supplementary material S1 and S2). Conversely, the non-UHR patients had significantly higher global functioning than the UHR patients. Participants were recruited between January 2017 and June 2019 and were drugnaïve at the time of the clinical assessment. The Ethics Committee of the Department of Dynamic and Clinical Psychology of the Sapienza University of Rome (No. 44/2017) approved the study.

\section{Measures}

\section{Psychological functioning and clinical diagnosis}

The level of functioning was measured with the Childhood Global Assessment Scale (Shaffer et al., 1983). Clinicians made psychiatric diagnoses according to the Structured Clinical Interview for the Diagnostic and Statistical Manual of Mental Disorders (5th ed.; DSM-5; APA, 2013).

\section{Attenuated psychotic symptoms}

Patients' UHR symptoms were indexed on the Structured Interview for Psychosis-Risk Syndrome version 5.3. (SIPS; McGlashan et al., 2013). The SIPS includes subscales for the ratings of 19 symptoms (five positive, six negative, four disorganized, and four general) that are evaluated based on the presence, duration, and severity of specific experiences and behaviors. Each of the five positive items is rated on a scale from 0 (no symptoms) to 6 (extreme or psychotic symptoms). A score of 3, 4, or 5 on at least one of the positive items (with specific requirements regarding onset and frequency) indicates the pres- ence of an UHR-state. Specifically, the measure contains diagnostic criteria for three psychosis-risk syndromes, i.e. Attenuated Positive Symptom psychosis-risk Syndrome (APSS); Brief Intermittent Psychosis-risk Syndrome (BIPS); and Genetic Risk and functional Decline (GRD, which is characterized by schizotypal personality disorder or first-degree familiarity with schizophrenia spectrum disorders and significant global functioning decline over a 12-month period). Finally, a score of 6 on any of the five positive items indicates the presence of a (full-blown) psychotic syndrome.

\section{Defense mechanisms}

The Defense Mechanism Rating Scales (DMRS; Perry, 1990; Perry, \& Henry, 2004) is an observer-rater instrument to assess the use of defense mechanisms based on verbatim transcripts of clinical interviews or psychotherapy sessions. DMRS qualitative scores reflect no use, probable use, and definite use of 30 specific defense mechanisms illustrated by examples and further rules (e.g., definition of each, a description of the intrapsychic function, and a list of similar mechanisms and indications of how to distinguish them). The scale also provides three quantitative scores: Overall Defensive Functioning (ODF), defense level scores, and individual defense scores. The ODF score was computed by adding the absolute frequency of defense mechanisms within each level (see below) and multiplying each subtotal by its specific weight, ranging from 1 to 7 . Defense level scores were calculated as the proportional (i.e., percentage) scores for each of the seven defense levels. Finally, individual defense scores are the proportional scores for each of the 30 defense mechanisms, weighted by the total instances of all defense mechanisms. DMRS defenses are ordered hierarchically into seven levels, according to Vaillant's (1992) conception of defensive functioning (Table 2). From the least to most adaptive, defense levels and their functions are as follow: i) action defenses (e.g., acting out, passive aggression, and helprejecting complaining), through which one acts or withdrawals to deal with internal or external stressors; ii) major image distortion defenses (e.g., splitting of self and others' image and projective identification), through which one eliminates ambivalence toward an object by distorting its image according to a single emotional view;

Table 1. Characteristics of sample means and standard deviations, and comparative test between groups (T-test and chi-square)

\begin{tabular}{lcccc}
\hline & $\begin{array}{c}\text { Total sample } \\
(\boldsymbol{N}=\mathbf{5 2}) \\
\boldsymbol{M} \mathbf{s d}\end{array}$ & $\begin{array}{c}\text { non-UHR } \\
(\boldsymbol{n}=\mathbf{2 6}) \\
\boldsymbol{M} \mathbf{s d}\end{array}$ & $\begin{array}{c}\text { UHR } \\
(\boldsymbol{n}=\mathbf{2 6}) \\
\mathbf{M} \mathbf{s d}\end{array}$ & \\
\hline Age & $15.58 \pm 1.23$ & $15.69 \pm 1.36$ & $15.46 \pm 1.37$ & $\mathrm{t}=-.642(\mathrm{p}=.52)$ \\
\hline Gender $(\mathrm{m} / \mathrm{f})$ & $21(40.4 \%) / 31(59.6 \%)$ & $9(28.9 \%) / 17(71.1 \%)$ & $12(46.2 \%) / 14(53.8 \%)$ & $\mathrm{X}^{2}=.719(\mathrm{p}=.397)$ \\
\hline Years of education & $9.98 \pm 1.53$ & $10.15 \pm 1.48$ & $9.81 \pm 1.57$ & $\mathrm{t}=-.814(\mathrm{p}=.42)$ \\
\hline GAF & $67.52 \pm 13.54$ & $75.89 \pm 8.63$ & $62.38 \pm 13.53$ & $\mathrm{Z}=1.63(\mathrm{p}=0.01)$ \\
\hline
\end{tabular}


iii) disavowal defenses (e.g., denial, projection, rationalization, and autistic fantasy), through which one avoids recognizing certain stressful aspects of reality that may appear clear to outside observers; iv) minor image distortion defenses (e.g., omnipotence, idealization of self and others' image, and devaluation of self and others' image), through which one distorts one's image of self, body, or others to dismiss problems and up-regulate selfesteem; v) neurotic defenses (e.g., repression, dissociation, reaction formation, and displacement), through which one maintains a state of unawareness with respect to conflicting needs, desires, and ideas associated with charged feelings, while still expressing these needs, desires, and ideas in symbolically meaningful ways; vi) obsessional defenses (e.g., undoing, intellectualization, and isolation of affects), through which one distances oneself from potentially threatening feelings while maintaining cognitive awareness and not distorting related stressors; vi) high adaptive defenses (e.g., affiliation, altruism, anticipation, humor, self-assertion, selfobservation, sublimation, and suppression), through which one demonstrates maximal adaptation to life and integration of feelings, ideas, and their consequences.

For the purpose of this study, the defense mechanism assessment was complemented by applying the P-DMRS (Berney et al., 2014), an observer-rater instrument that integrates the DMRS with an additional level of defense. Valliant (1971) originally described three psychotic defense mechanisms (delusional projection, psychotic denial, and distortion) that have been subsequently listed in the optional axis for a hierarchy of defenses of DSMIV (APA, 1994). The APA (1994) included these defenses in the "level of defensive dysregulation," which is characterized by failure of defensive regulation to contain an individual's reaction to stressors, leading to a pronounced break with objective reality. The P-DMRS expanded this taxonomy by providing definitions, functions, discriminations, and clinical examples of six defense mechanisms considered "psychotic": psychotic denial, autistic withdrawal, distortion, delusional projection, fragmentation, and concretization (for a detailed description of each defense mechanism reported in the manual, see Table 3). In the ODF calculation, psychotic defenses were considered level 0 , yielding a final score between 0 (lowest) and 7 (highest).

\section{Procedure}

In the first step, all clinical and demographic data were collected, and clinical diagnoses were made according to DSM-5 criteria. Subsequently, two research assistants who had attended specific training interviewed all patients with the SIPS and, 2-3 weeks later, with the Adult Attachment Interview (AAI; George, Kaplan, \& Main, 1984, 1996). All AAI interviews were audiorecorded and transcribed. Blind to the other study variables, two of the authors (G. L. and S. S.) coded DMRS and P-DMRS data from the AAI interview transcripts (they had undergone a 50-h training program delivered by an expert DMRS judge). Reliability coefficients on $50 \%$ of the ratings were established and yielded satisfactory results in terms of intraclass correlation coefficients between 0.67 and 0.91 .

\section{Data Analysis}

A $t$-test was applied to evaluate the differences between UHR and non-UHR patients in terms of their use of defense mechanisms. Such differences were computed using each single defense mechanism, cluster (ranging from psychotic to mature defenses) and ODF scores. Single defense mechanisms and clusters were calculated according to "proportional defense scoring." Specifically, to account for the variability between subjects (i.e., participants varied in how many defenses they used), the raw frequency score of each defense (e.g., 0.1 or 2) was divided by the total sum of all defenses (proportion) and multiplied by 100 , yielding a percentage score (see the methods section). Spearman's correlations were calculated to estimate putative associations between single defense mechanisms and subthreshold psychotic symptoms (i.e., positive, negative, and general). All analyses were conducted using SPSS (Version 25) for Mac.

Table 2. Hierarchy of Defense Levels and Individual Defense Mechanisms according to DMRS and P-DMRS

\begin{tabular}{ll}
\hline Levels & Defense mechanisms \\
\hline 7 Highly adaptive (mature) defenses & Affiliation, altruism, anticipation, humor, self-assertion, self-observation, sublimation, suppression \\
\hline 6 Obsessional defenses & Isolation of affect, intellectualization, undoing \\
\hline 5 Neurotic defenses & Repression, dissociation, reaction formation, displacement \\
\hline 4 Minor image-distorting (narcissistic) defenses & Devaluation (of self and others' images), idealization (of self and others' images), omnipotence \\
\hline 3 Disavowal defenses & Denial, projection, rationalization, autistic fantasy \\
\hline 2 Major image-distorting (borderline) defenses & Splitting (of self and others' images), projective identification \\
\hline 1 Action defenses & Acting out, help-rejecting, complaining, projective identification \\
\hline 0 Psychotic defenses & Psychotic denial, autistic withdrawal, distortion, delusional projection, fragmentation, concretization \\
\hline
\end{tabular}

DMRS = Defense Mechanisms Rating Scales; P-DMRS = Psychotic-Defense Mechanisms Rating Scales. 


\section{Results}

\section{Defense Specificities of UHR patients}

Overall, UHR patients showed a significantly less amount of defense mechanisms performed (i.e., total instances of defense mechanisms observed) compared with controls $(t=2.376 ; p=.02)$. Moreover, ODF was lower in UHR patients compared with controls $(t=-2.451 ; p=$ $.02)$. No between-group differences were observed regarding defense mechanism levels, except for psychoticlevel defenses, which UHR individuals performed more frequently $(t=1.925 ; p=.06)$. Regarding between-group differences in single defense mechanisms, fragmentation was the sole defense which has been exclusively performed by UHR individuals $(t=1.971 ; p=.05)$. Effect sizes of the reported between-group differences were large, except for "number of defenses" where medium effect size was observed (Table 4).

\section{Associations Between Defense Mechanisms and Subthreshold Psychotic Symptoms}

Results showed significant, positive, and strong correlations between psychotic-level defenses and negative symptoms of social anhedonia $(r=.630 ; p<.01)$, avolition $(r=.504 ; p<.01)$, expression of emotions $(r=.510$; $p<.01)$, and experience of emotion and self $(r=.500 ; p$ $<.01)$. Positive and significant correlations were observed between psychotic defenses and disorganization symptoms of odd behavior or appearance $(r=.536 ; p<.01)$ and bizarre thinking $(r=.430 ; p=.02)$. Finally, mature level defenses were negatively correlated with unusual thought content or delusional ideas $(r=-.464 ; p=.01)$.
Effect sizes of the significant correlations were large, except for unusual thought content and bizarre thinking, where medium effect sizes were observed (Table 5).

\section{Discussion}

The main aims of the present study were to investigate the defense mechanisms used by UHR versus non-UHR patients to shed light on the particular mechanisms theoretically involved in the development of psychosis and to add information about the associations between defense mechanisms and dimensional markers of the UHR state (i.e., subclinical psychotic symptoms).

With regard to the latter, a nonlinear relationship between psychotic-level defense mechanisms and attenuated psychotic symptoms was observed. In particular, psychotic defenses were strongly associated with negative symptoms, whereas no association with positive symptoms was found. Various explanations might account for the mixed findings. First, we can assume that the maladaptive strategies in dealing with internal and external stressor that refer to psychotic defense mechanisms may be involved in the expression of negative symptoms' dimensions, such as avolition-apathy (social anhedonia and avolition items on the SIPS) and expressive deficit ("expression of emotions" and "experience of emotion and self' items on the SIPS). Negative symptoms, in turn, may interact with positive symptoms, accounting for the wellknown covariation between them that has been frequently observed in psychotic samples (Borsboom \& Cramer, 2013). Second, positive and negative symptoms could be independent of each other (Carrà et al., 2019; Galderisi,

Table 3. P-DMRS: Definitions and Functioning.

\begin{tabular}{|c|c|}
\hline Defense & Definition and Functioning \\
\hline Denial & $\begin{array}{l}\text { The main defense mechanism that may underlie the psychotic experience (Seltzer et al., 1989), denial minimizes distress by ac- } \\
\text { tively refusing to recognize aspects of internal experience (neurotic denial) or internal and external experience (psychotic denial; } \\
\text { Berney et al., 2013; Hauser, 1986). }\end{array}$ \\
\hline Delusional projection & $\begin{array}{l}\text { Delusional projection involves persecutory delusion with no reality testing (Selzer, Sullivan, Carsky, \& Terkelsen, 1989). Subjects } \\
\text { may attribute their feelings to others or perceive the presence of others within themselves (e.g., psychotic depression; Vaillant, } \\
\text { 1992) }\end{array}$ \\
\hline Fragmentation & $\begin{array}{l}\text { A person's representations of self and others (and their connections) are fragmented in multiple ways, leading to an active and } \\
\text { pervasive division of various aspects of the experience. It can be considered active disorganization, leading to a confused and } \\
\text { disorganized narrative (Berney et al., 2013). Subjects who use this defense appear confused, and their narration of even a simple } \\
\text { theme can be challenging for interlocutors to understand }\end{array}$ \\
\hline Concretization & $\begin{array}{l}\text { Concretization refers to the transformation of a mental representation into a concrete object, situation, or action. The choice of } \\
\text { the concrete form into which a certain mental representation is transformed is not at all random but symbolically connected to its } \\
\text { internal and abstract representation }\end{array}$ \\
\hline Autistic withdrawal & $\begin{array}{l}\text { External and internal stressors are removed from the subject's experience by means of disconnection from the environment; this } \\
\text { defense is characterized by a lost sense of continuous experience, and it can be clinically identified by a patient's behavior, rather } \\
\text { than his or her verbal conveyances (Berney et al., 2013) }\end{array}$ \\
\hline Distortion & $\begin{array}{l}\text { A subject can react to stressors by altering external or internal reality, distorting objects into something to which the user can } \\
\text { react. Distortion can be manic, depressive, or structured (whereby a new reality is constructed with narrative coherence). Distorted } \\
\text { reality has the function of bestowing a sense of omnipotence; it protects the subject's self-esteem but alters reality in a delirious } \\
\text { way, with reality treated as a part of the self (Berney et al., 2013) }\end{array}$ \\
\hline
\end{tabular}


Table 4. Differences in defense mechanisms between UHR and non-UHR individuals.

\begin{tabular}{|c|c|c|c|c|c|c|c|}
\hline & \multicolumn{2}{|c|}{ UHR (n=26) } & \multicolumn{2}{|c|}{ non-UHR $(n=26)$} & \multirow[b]{2}{*}{ T-test } & \multirow[b]{2}{*}{$\mathbf{P}$} & \multirow[b]{2}{*}{ Cohen's D } \\
\hline & M & SD & M & SD & & & \\
\hline Num. defenses & 44.65 & $(21.40)$ & 59.62 & $(21.42)$ & $-2.52 *$ & .015 & Medium \\
\hline Overall Defensive Functioning (ODF) & 3.9 & $(0.46)$ & 4.18 & $(0.35)$ & $-2.45^{*}$ & $<.05$ & Large \\
\hline DMRS Defenses & $\mathrm{M} \%$ & $\mathrm{SD} \%$ & $\mathrm{M} \%$ & $\mathrm{SD} \%$ & T-test & $\mathrm{P}$ & Cohen's D \\
\hline 7. High-adaptive & 1.45 & $(2.72)$ & 2.41 & $(3.64)$ & -1.08 & 0.29 & \\
\hline Affiliation & 0.19 & $(0.82)$ & 0.14 & $(0.71)$ & 0.26 & 0.80 & \\
\hline Altruism & 0.25 & $(0.92)$ & 0.07 & $(0.36)$ & 0.95 & 0.35 & \\
\hline Anticipation & 0.08 & $(0.40)$ & 0.0 & $(0.0)$ & 1.00 & 0.32 & \\
\hline Humor & 0.30 & $(0.91)$ & 1.27 & $(1.94)$ & $-2.30^{*}$ & $<.05$ & Large \\
\hline Self-observation & 0.62 & $(1.18)$ & 0.75 & $(1.78)$ & -0.31 & 0.76 & \\
\hline Self-assertion & 0.0 & $(0.0)$ & 0.0 & $(0.0)$ & B & $\mathrm{B}$ & \\
\hline Sublimation & 0.0 & $(0.0)$ & 0.0 & $(0.0)$ & $\mathrm{B}$ & B & \\
\hline Suppression & 0.0 & $(0.0)$ & 0.18 & $(0.93)$ & -1 & 0.32 & \\
\hline 6. Obsessional & 10.62 & $(8.71)$ & 13.18 & $(7.16)$ & -1.16 & 0.25 & \\
\hline Isolation of affect & 0.95 & $(1.52)$ & 1.33 & $(1.72)$ & -0.84 & 0.40 & \\
\hline Intellectualization & 7.99 & $(8.07)$ & 9.32 & $(6.88)$ & -0.64 & 0.53 & \\
\hline Undoing & 1.68 & $(2.57)$ & 2.53 & $(2.82)$ & -1.14 & 0.26 & \\
\hline 5. Neurotic & 32.73 & $(13.31)$ & 35.08 & $(11.82)$ & -0.67 & 0.51 & \\
\hline Repression & 26.67 & $(15.00)$ & 27.88 & (12.87) & -0.31 & 0.76 & \\
\hline Dissociation & 1.26 & $(2.00)$ & 1.1 & $(1.80)$ & 0.29 & 0.78 & \\
\hline Reaction formation & 2.19 & $(2.72)$ & 2.05 & $(1.91)$ & 0.21 & 0.84 & \\
\hline Displacement & 2.62 & $(3.08)$ & 4.03 & $(3.70)$ & -1.50 & 0.14 & \\
\hline 4. Narcissistic & 12.73 & $(7.53)$ & 14.77 & $(8.17)$ & -0.94 & 0.35 & \\
\hline Devaluation & 6.05 & $(4.45)$ & 7.05 & $(5.79)$ & -0.70 & 0.49 & \\
\hline Idealization & 5.50 & $(5.89)$ & 5.87 & $(5.62)$ & -0.24 & 0.82 & \\
\hline Omnipotence & 1.19 & $(2.31)$ & 1.84 & $(3.64)$ & -0.78 & 0.44 & \\
\hline 3. Disavowal & 27.95 & $(6.70)$ & 24.43 & $(8.65)$ & 1.64 & 0.11 & \\
\hline Denial & 13.29 & $(8.30)$ & 9.65 & $(5.58)$ & 1.86 & 0.07 & \\
\hline Projection & 2.00 & $(2.88)$ & 1.58 & $(1.62)$ & 0.65 & 0.52 & \\
\hline Rationalization & 9.35 & $(5.47)$ & 10.68 & $(4.60)$ & -0.95 & 0.35 & \\
\hline Autistic fantasy & 3.31 & $(3.12)$ & 2.52 & $(2.51)$ & 1.01 & 0.32 & \\
\hline 2. Borderline & 5.46 & $(7.16)$ & 4.82 & $(5.23)$ & 0.37 & 0.72 & \\
\hline Splitting & 5.06 & $(6.76)$ & 4.48 & $(5.09)$ & 0.35 & 0.73 & \\
\hline Projective identification & 0.40 & $(0.94)$ & 0.34 & $(0.85)$ & 0.25 & 0.81 & \\
\hline$\underline{\text { 1. Action }}$ & 7.07 & $(6.08)$ & 4.64 & $(5.17)$ & 1.55 & 0.13 & \\
\hline Acting out & 0.46 & $(1.20)$ & 0.11 & $(0.39)$ & 1.43 & 0.16 & \\
\hline Passive aggression & 6.30 & $(6.39)$ & 4.46 & $(5.12)$ & 1.15 & 0.26 & \\
\hline Hypochondriasis & 0.31 & $(1.38)$ & 0.08 & $(0.28)$ & 0.83 & 0.41 & \\
\hline 0. Psychotic & 2.00 & $(3.24)$ & 0.68 & $(1.36)$ & 1.93 & 0.06 & \\
\hline Psychotic denial & 0.33 & $(0.91)$ & 0.09 & $(0.45)$ & 1.22 & 0.23 & \\
\hline Autistic withdrawal & 0.22 & $(0.77)$ & 0.0 & $(0.0)$ & 1.44 & 0.16 & \\
\hline Distortion & 0.7 & $(1.67)$ & 0.39 & $(1.01)$ & 0.79 & 0.43 & \\
\hline Delusional projection & 0.14 & $(0.51)$ & 0.20 & $(0.70)$ & -0.32 & 0.75 & \\
\hline Fragmentation & 0.47 & $(1.22)$ & 0.0 & $(0.0)$ & 1.97 & 0.05 & n.c.** \\
\hline Concretization & 0.14 & $(0.73)$ & 0.0 & $(0.0)$ & 1 & 0.32 & \\
\hline
\end{tabular}

* Cohen's d Effect size ranges: Very small 0.01; Small 0.20; Medium 0.50; Large 0.80; Very large 1.20; Huge 2.0 (Cohen, 1988; Sawilowsky, 2009).

$* *$ n.c. not calculable because presence $v s$. complete absence. 
Mucci, Buchanan, \& Arango, 2018), and the association between psychotic defenses and negative symptoms may be explained by a common cause, the most obvious candidate being neurocognitive impairments (de Gracia Dominguez, Viechtbauer, Simons, van Os, \& Krabbendam, 2009; Gur et al., 2015; Leanza et al., 2018; Meyer et al., 2014). Nevertheless, due to the cross-sectional design and the small sample size of the present study, causal inferences would be furthered by research using other designs and different data analysis.

With regard to the defense specificities of UHR individuals, a specific defense vulnerability was observed. First, compared to non-UHR individuals, UHR patients showed a significantly lower ODF. Second, the number of defense mechanisms (i.e., total instances of defense mechanisms observed) was significantly lower in UHR individuals, pointing to the patients' overall minor production of defenses. Overall, if we consider defense mechanisms as 'adjustment' strategies, the findings suggest that UHR patients are less able to cope adaptively, which offers a different perspective on the emotional and symptomatic reactivity to daily life stress previously observed in UHR individuals (Gerritsen et al., 2019; Palmier-Claus, Dunn, \& Lewis, 2012; van der Steen et al., 2017). Finally, psychoticlevel defenses are presented more often in UHR individuals compared to non-UHR patients (although this result does not reach high statistical significance), whereas no substantial differences were found with respect to other defense levels or single defenses. UHR patients were quantitatively less productive in terms of defense or adjustment mechanisms and qualitatively more impaired in their functioning. Among the psychotic defenses, fragmentation seems a better way to discriminate between UHR and non-UHR patients; hence, this specific defense mechanism may be a marker of UHR patients' psychological functioning.
In the psychoanalytic literature, fragmentation has been described as a defense mechanism with both a specific behavioral manifestation and, more importantly, certain consequences to mental functioning and one's subjective experience of self. Regarding the first aspect, the behavioral manifestation is mostly traced in disorganized and confusing narratives as a result of disorganized thought (Berney et al., 2014). In this regard, fragmentation can be considered active disorganization (Vaillant, 2011). Indeed, it is not surprising that in the present study psychotic defenses were positively correlated with disorganization symptoms such as bizarre thinking and odd behavior or appearance. Concerning its effect on mental functioning and subjective experience, psychoanalysts have linked fragmentation to a dissolution of self that could severely impair both the consistency of mental representations (i.e., internal objects) and one's sense of personal identity. In particular, fragmentation is achieved through repeated splitting of the self and other representations, leading to a disintegration of the self and independent and uncontrolled existence, as well as projection onto others (e.g., one's therapist) or inanimate objects ${ }^{2}$ (Arieti, 1974; Bion, 1967); Klein, 1946). Consequently, Resnik (1986) characterized the psychotic break by a loss of "uniqueness" and personal identity, which involves disconnectedness among the projected parts of the self, rendering it fragmented and depleted. Overall, it seems that all psychodynamic formulations of fragmentation, as well as other psychotic defenses, describe primary anxiety driven by the breakdown of the self and the dissolution of psychic structure, albeit in different ways (Munich, 1995). Psychodynamic descriptions of subjective correlates in patients' experiences of psychotic defense mechanisms show important similarities with the so-called basic or "minimal" self-awareness disorder recently described and

Table 5. Correlations Between Defense Mechanisms and Subthreshold Psychotic Symptoms.

\begin{tabular}{|c|c|c|c|c|c|c|c|c|}
\hline & Psychotic & Action & Borderline & Disavowal & Narcissistic & Neurotic & Obsessional & Mature \\
\hline P1 Unusual Thought Content/Delusional Ideas & .26 & .14 & 0.01 & -0.11 & -0.16 & 0.10 & -0.23 & $-.46^{*}$ \\
\hline P2 Suspiciousness/Persecutory Ideas & .07 & -.12 & -.13 & -.10 & -0.33 & .27 & -.05 & -.10 \\
\hline P3 Grandiose ideas & .01 & .24 & -.03 & -.01 & -.04 & -.13 & -.12 & .05 \\
\hline P4 Perceptual Abnormalities/Hallucinations & -.01 & -.10 & -.18 & -.17 & .04 & -.06 & -.00 & .01 \\
\hline P5 Disorganized Communication & -.01 & 0.16 & -0.34 & -.20 & .16 & -.07 & .00 & .05 \\
\hline Total P & .14 & .11 & -.18 & -.19 & -.05 & -.02 & -.12 & -.14 \\
\hline N1 Social Anhedonia & $.63 * *$ & -.07 & -.12 & -.01 & -.07 & -.03 & -.21 & -.13 \\
\hline N2 Avolition & $.50 * *$ & -.22 & .02 & -.09 & -.11 & .06 & -.33 & -.08 \\
\hline N3 Expression of Emotions & $.51 * *$ & -.26 & -.19 & -.24 & -.05 & .04 & -.03 & .00 \\
\hline N4 Experience of Emotion and Self & $.50 * *$ & -.27 & -.27 & -.23 & .03 & .07 & -.13 & -.03 \\
\hline N5 Ideational Richness & .36 & .10 & .08 & -.03 & -.09 & -.05 & -.15 & -.02 \\
\hline N6 Occupational Functioning & .35 & -.33 & -.00 & -.26 & .05 & .23 & -.28 & -.07 \\
\hline Total N & $.49^{*}$ & -.29 & -.22 & -.21 & -.14 & .14 & -.11 & .02 \\
\hline D1 Odd Behavior of Appearance & $.54 * *$ & 0.01 & -.05 & .03 & 0.03 & -.09 & -.33 & -.16 \\
\hline D2 Bizarre Thinking & $.43^{*}$ & -.10 & .06 & -.07 & -.02 & -.05 & -.26 & -.08 \\
\hline D3 Trouble with Focus and Attention & .13 & .11 & -.20 & .05 & .12 & -.00 & -.22 & -.25 \\
\hline D4 Impairment in Personal Hygiene & .29 & .14 & .20 & -.11 & .08 & -.12 & -.30 & -.18 \\
\hline Total D & $.39 *$ & -.02 & .00 & -.07 & .09 & -.09 & -.28 & -.15 \\
\hline
\end{tabular}

Pearson's r Effect size: * medium $r>.30 ; * *$ large $r>.50(\text { Cohen, } 1988)^{55}$ 
operationalized within the phenomenological framework; this disorder describes a set of experiential or subjective anomalies that occur in schizophrenia and no other mental disorder (Parnas et al., 2005; Sass \& Parnas, 2003). Among these anomalous experiences, a diminished sense of basic self describes a pervasive sense of inner void, a lack of an inner nucleus, a pervasive lack of identity, and a feeling of being anonymous, as if the individual does not exist. Some phenomenological descriptions also describe the subjective experience of a massive projection of split-off parts of the self. For example, two items on the Examination of Anomalous Self-Experience scale (Parnas et al., 2005) describe both identity confusion and the so-called "I-split" (i.e., ich-spaltung), in which "the patient experiences his I, self, or person as being divided or otherwise compartmentalized, disintegrated into semiindependent parts, or not existing as one unified whole" (Parnas et al., 2005, p. 248). ${ }^{3}$ It seems that the psychoanalytic and phenomenological investigations, irrespective of their epistemological differences (for a review, see Molaro, 2016), focus on the inability of psychotic individuals to perceive a consolidated, integrated, and individualized sense of self.

Nevertheless, a major divergence between these two theoretical frameworks concerns how self-disorders should be considered. In particular, it is not clear whether self-disorders should be better understood as an outcome of certain defense mechanisms or as a primary phenomenon. In the psychoanalytic framework, self-disorders are considered a consequence of a dynamic process in which a sequence of specific defenses (e.g., splitting, projection, and projective identification) are repeatedly mobilized against the threat of psychic annihilation, thereby creating a state of fragmentation (Costantinides \& Back, 2010). Therefore, in the psychoanalytic explanation, psychotic defenses are defined as mental processes that are actively (though unconsciously) mobilized by the subject to mitigate the distressing effect of certain emotions or mental representations, eventually leading to a disintegration of the self (i.e. anomalous self-experiences). Conversely, in the phenomenological framework, anomalous self-experiences are considered a result of a primary and fundamental phenomenon; that is, the disturbance of "minimal self," which refers to a fragility in implicit first-person perspective, presence, and agency, resulting in a trait-like alteration of the very structure of consciousness ${ }^{4}$ (Gallagher, 2011; Parnas, 2012; Sas et al., 2018). This theoretical divergence between the psychoanalytic and phenomenological framework in understanding self-disorders could also rely on different definitions of the concept of self. Although in the psychoanalytic literature the concept of self is often referred to as "the integration of representations of the self" (e.g., Kernberg, 2016), in contemporary phenomenology and cognitive science an important distinction is made between the "narrative" self (i.e., self as a theme or object of conscious awareness and attention) and the "minimal" or "core/basic" self (i.e., self as a tacit structure of experience that is a prerequisite of the narrative self; e.g., Damasio, 1999; Gallagher, 2000; Gallagher \& Zahavi, 2012; Zahavi, 2005). It seems psychodynamic scholars still need to address the empirically grounded distinction between "narrative" and "basic" self. As Zandersen \& Parnas (2018) recently asked, "Does the [psychoanalytic] self-concept refer to the person's beliefs about him-/herself, which can be linguistically expressed and thematized? Or is it a sub-personal, unconscious, dispositional structure that only occasionally becomes materialized as a belief about oneself, e.g. through psychotherapy?" (p. 4). For example, it could be argued that some defense mechanisms (e.g., splitting, projection, or projective identification) can affect internal representations by hesitating in a disorder of "narrative self" (e.g., identity diffusion; Kernberg, 1975; Kernberg, 1984; Kernberg, 2016). However, deeper disorganized mental states (e.g., fragmentation) can be better understood as a result of primary and structural disorder of the "basic" self, rather than an outcome of psychotic defense mechanisms. Psychodynamic taxonomy of psychotic defense mechanisms, as well as clinical descriptions of self-disorders formulated by eminent psychoanalytic scholars (e.g., Bion 1959, 1967; Klein, 1946), may benefit from future integration with recent developments of neuroscience, philosophy of mind, and phenomenology (e.g., Gallagher \& Zahavi, 2012; Nelson et al., 2020; Sas et al., 2018).

Bion (1967) extensively described fragmentation as a process through which a psychotic individual breaks down his or her thoughts, perceptions, and feelings into smaller and more manageable parts. It is considered a typical phenomenon of Klein's (1946) so-called “paranoidschizoid position" by which i) one's representations of self and others are massively split off; ii) one's sadistic and destructive wishes are projected outside the self; and iii) anxiety associated with threatening objects (which contain the projections of such sadistic and destructive wishes) mostly characterizes the emotional experience.

Similarly, Bion (1967), in his conceptualization of psychotic fragmentation, introduced the notion of "unmentalized sense impressions" (i.e., beta-elements). In his developmental model, Bion linked the ability to form self-concepts (e.g., the sense that one has a mind and a personal existence) to the capability to internalize a specific mental function (the so-called "alpha function," which is conceptually similar to the contemporary concept of "mentalization"). A caregiver provides this capability early in development, which enables an individual to contain and transform perceptions into cognitive contents (or alpha-elements) that can be used to think, remember, and dream (Jackson, 2002).

4 This formulation seems to be supported by recent finding that pointed to specific neurocorrelates (neurocognitive, neurophysiological, and neurobiological correlates) of minimal self-disturbances. In particular, it has been observed that the neuroconstruct of source-monitoring deficits (i.e., difficulties in making attributions about the origins of mental experiences, e.g., whether an experience was real or imagined, or whether its origin was internal [self-generated] or external [other-generated]) explained $39.8 \%$ of the variance in Examination of Anomalous Self-Experience scores, showing an increasing gradient of severity from healthy control participants to high-risk patients to first-episode psychosis patients (first-episode psychosis $<$ high risk $<$ healthy control; Nelson et al., 2019; Nelson et al., 2020). 


\section{Conclusions}

UHR individuals show an overall impairment in defensive functioning (ODF), a minor production of defenses, and a higher production of psychotic-level defenses. The findings have relevant implications with regard to psychotherapeutic treatment for UHR individuals. The goal of addressing defenses in the therapeutic process is to help patients make sense of their personal experiences and develop more adaptive responses to emotionally difficult situations (Lingiardi \& McWilliams, 2017; Rosenbaum et al., 2012). In this vein, defenses also seem relevant to the ability of the psychotherapeutic process to help patients understand their feelings, attitudes, and subjective intentions in a real interpersonal relationship. Such therapeutic work aims at developing patients' adaptive mental functioning when dealing with emotional experiences to improve their ability to integrate affects and internal representations. Previous findings showed that addressing or interpreting defenses in the psychotherapeutic process improves defensive functioning within and across sessions (Perry, \& Bond, 2012; Winston, Winston, Samstag, \& Mu- ran, 1994) and that change in defense functioning can in turn mediate improvement in symptoms (Kramer, Despland, Michel, Drapeau, $\&$ de Roten, 2010). In this regard, the present investigation showed an inverse relationship between positive psychotic symptoms and adaptive defenses, as well as a positive relationship between psychotic defenses and negative symptoms. Moreover, evidence suggests that a patient's ability of work through and reflect on his or her defenses is also related to a strengthening of the patient's psychological capacities, and especially an increase in his or her ability to mentalize (e.g., Boldrini et al., 2018; Hörz-Sagstetter, Mertens, Isphording, Buchheim, \& Taubner, 2015; Levy et al., 2006). It is known that UHR individuals, as patients with schizoid and schizotypal personality disorders (Nazzaro et al., 2017), are characterized by impairment in social cognition (Armando, Hutsebaut \& Debbane, 2019; Bora \& Pantelis, 2013). Consequently, psychotherapeutic interventions that address the specific defense vulnerability of UHR patients could be effective in reducing symptom expression, promoting clinically significant changes in mentalizing abilities, and improving patients' long-term clinical outcomes.

Moreover, our findings showed that the early detection of psychotic defense mechanisms could improve screening methods for UHR patients. Indeed, only psychotic-level defenses discriminated between UHR and non-UHR patients in the present sample, so it is reasonable to assume that a partial and less timeconsuming assessment of defenses with the P-DMRS (instead of the full evaluation with the DMRS) could improve screening methods for the UHR status. Nevertheless, further research is needed to establish whether the P-DMRS can corroborate the evaluation of psychosis risk.
To the best of our knowledge, the present study was the first investigation of defense mechanisms in helpseeking patients at risk for psychosis. The results shed light on the role of defense mechanisms in the expression of subthreshold psychotic symptoms and opens up assessment and theoretical questions about the qualitative and quantitative differences between psychotic and nonpsychotic experiences. The study has several limitations. The present investigation is the first attempt, as far as we know, at performing DMRS coding on transcripts of AAI interviews. However, scholars who have developed both DMRS and P-DMRS make it clear that these tools can be applied to "dynamic interview transcripts," in which patients are asked to talk about their current lives and significant past episodes. In this regard, although the AAI's focus is on childhood experiences of attachment, it is nevertheless a standardized dynamic semi-structured interview, in which the subjects are invited to describe both past and current interpersonal experiences and to reflect on the narratives they have presented. From this point of view, the mainframe of the patients' information is the same for each participant. Second, participants in the control group were recruited from a clinical population; thus, the lack of between-group differences in defenses may be inflated by this methodological choice. For example, the implementation of borderline and action-level defenses by UHR patients could have been more evident if they had been compared with healthy subjects. Nevertheless, overreliance on healthy control participants in UHR research has been criticized (Millman, Gold, Mittal, \& Schiffman, 2019). Because UHR individuals are characterized by high comorbidity with nonpsychotic mental disorders (e.g., Boldrini et al., 2019; Fusar-Poli et al., 2012), enrolling clinical controls may be a better way to interpret between-group differences as a unique function of a psychosis-spectrum process.

\section{References}

American Psychiatric Association (2013) Diagnostic and statistical manual of mental disorders (5th ed). Arlington, VA: Author.

American Psychiatric Association. (1994). Diagnostic and statistical manual of mental disorders (4th ed.). Washington, DC: American Psychiatric Press.

Andreou, C., Bailey, B., Borgwardt S. (2019). Assessment and treatment of individuals at high risk for psychosis. BJPsych Advances 25, 177-184.

Armando, M., Hutsebaut, J., \& Debbane, M. (2019). A Mentalization-Informed Staging Approach to Clinical High Risk for Psychosis. Frontiers in Psychiatry, 10.

Babl, A., Holtforth, M.G., Perry, J.C., Schneider, N., Dommann, E., Heer, S., Stähli, A., Aeschbacher, N., Eggel, M., Eggenberg, J., Sonntag, M., Berger, T., Caspar, F. (2019). Comparison and changes of defense mechanisms over the course of psychotherapy in patients with depression or anxiety disorder: Evidence from a randomized controlled trial. Journal of Affective Disorders, 252, 212-220. 
Berney, S., de Roten, Y., Beretta, V., Kramer, U., \& Despland, J. N. (2014). Identifying psychotic defenses in a clinical interview. Journal of Clinical Psychology: In Session, 70(5), 428-439.

Bion, W. R. (1959). Attacks on linking. International Journal of Psychoanalysis, 40, 308-315.

Bion, W. R. (1967). Second Thoughts. London, Karnac Books, 1984.

Boldrini, T., Nazzaro, M. P., Damiani, R., Genova, F., Gazzillo, F., \& Lingiardi, V. (2018). Mentalization as a predictor of psychoanalytic outcome: An empirical study of transcribed psychoanalytic sessions through the lenses of a computerized text analysis measure of reflective functioning. Psychoanalytic Psychology, 35(2), 196-204.

Boldrini, T., Tanzilli, A., Pontillo, M., Chirumbolo, A., Vicari, S., \& Lingiardi, V. (2019). Comorbid Personality Disorders in Individuals with an At-Risk Mental State for Psychosis: A Meta-Analytic Review. Frontiers in Psychiatry, 10:429.

Bora, E., \& Pantelis, C. (2013). Theory of mind impairments in first-episode psychosis, individuals at ultra-high risk for psychosis and in first-degree relatives of schizophrenia: Systematic review and meta-analysis. Schizophrenia Research, 144(1-3), 31-36.

Borsboom, D., \& Cramer, A. O. (2013). Network analysis: an integrative approach to the structure of psychopathology. Annual Review of Clinical Psychology, 9, 91-121.

Carrà, G., Crocamo, C., Angermeyer, M., Brugha, T., Toumi, M., \& Bebbington, P. (2019). Positive and negative symptoms in schizophrenia: A longitudinal analysis using latent variable structural equation modelling. Schizophrenia Research, 204, 58-64.

Cichocki, L. (2008). Changes in defence mechanisms of people suffering from psychotic disorders and receiving therapy in the Day Treatment Centre. Archives of Psychiatry and Psychotherapy, 4, 27-32.

Cramer, P. (1998). Coping and defense mechanisms: What's the difference? Journal of Personality, 66(6), 919-946.

Cramer, P. (2000). Defense mechanisms in psychology today. Further processes of adaptation. American Psychologist, 55(6), 637-646.

Cramer, P. (2009). The development of defense mechanisms from preadolescence to early adulthood: do IQ and social class matters? A longitudinal study. Journal of Research in Personality, 43,464-471.

Damasio A. R. (1999). The Feeling of What Happens: Body and Emotion in the Making of Consciousness. New York, NY: Harcourt Brace.

de Gracia Dominguez, M., Viechtbauer, W., Simons, C.J., van Os, J., Krabbendam, L. (2009). Are psychotic psychopathology and neurocognition orthogonal? A systematic review of their associations. Psychological Bullettin, 135, 157.

Di Giuseppe, M., Di Silvestre, A., Lo Sterzo, R., Hitchcott, P., Gemignani, A., Conversano, C. (2019a). Qualitative and quantitative analysis of the defense profile in Breast Cancer women: A pilot study. Health Psychology Open, 6(1), 1-6.

Di Giuseppe, M., Gennaro, A., Lingiardi, V., \& Perry, J. C. (2019b). The role of defense mechanisms in emerging personality disorders in clinical adolescents. Psychiatry: Interpersonal and Biological Processes, 82(2), 128-142.

Di Giuseppe, M., Perry, J.C., Petraglia, J., Janzen, J., \& Lingiardi, V. (2014). Development of a Q-sort version of the Defense Mechanism Rating Scales (DMRS-Q) for clinical use. Journal of Clinical Psychology, In Session, 70, 452-465.
Euler, S., Stalujanis, E., Allenbach, G., Kolly S., de Roten, Y., Despland, N.J., \& Kramer, U. (2018). Dialectical behavior therapy skills training affects defense mechanisms in borderline personality disorder: An integrative approach of mechanisms in psychotherapy. Psychotherapy Research 29(8), 1074-1085.

Foto-Özdemir, D., Akdemir, D., Çuhadaroglu-Çetin, F. (2016). Gender differences in defense mechanisms, ways of coping with stress and sense of identity in adolescent suicide attempts. Turkish Journal of Pediatry 58, 271-281.

Galderisi, S., Mucci, A., Buchanan, R. W., \& Arango, C. (2018). Negative symptoms of schizophrenia: New developments and unanswered research questions. The Lancet Psychiatry 5(8), 664-677.

Gallagher, S. (2000). Philosophical Conceptions of the Self: Implications for Cognitive Science. Trends in Cognitive Sciences 4(1), 14-21.

Gallagher, S. (Ed.). (2011). The oxford handbook of the self. Oxford, Oxford University Press.

Gallagher, S., \& Zahavi, D. (2012). The Phenomenological Mind. 2nd ed. Abingdon, UK: Routledge.

George, C., Kaplan, N., \& Main, M. (1984). The Berkeley Adult Attachment Interview. Unpublished manuscript. Department of Psychology, University of California, Berkeley.

Gerritsen, C., Bagby, R. M., Sanches, M., Kiang, M., Maheandiran, M., Prce, I., \& Mizrahi, R. (2019). Stress precedes negative symptom exacerbations in clinical high risk and early psychosis: A time-lagged experience sampling study. Schizophrenia Research, 210, 52-58.

Gur, R.E., March, M., Calkins, M.E., Weittenhiller, L., Wolf, D.H., Turetsky, B.I., Gur, R.C. (2015). Negative symptoms in youths with psychosis spectrum features: com- plementary scales in relation to neurocognitive performance and function. Schizophrenia Research, 166, 322-327.

Hauser, S. T. (1986). Conceptual and empirical dilemmas in the assessment of defences. In G. E. Vaillant (Ed.), Empirical studies of ego mechanisms of defense (pp. 89-100). Washington, DC: American Psychiatric Press.

Hilsenroth, M. J., Katz, M., \& Tanzilli, A. (2018). Psychotherapy research and the psychodynamic diagnostic manual (PDM2). Psychoanalytic Psychology, 35(3), 320-327.

Hörz-Sagstetter, S., Mertens, W., Isphording, S., Buchheim, A., $\&$ Taubner, S. (2015). Changes in reflective functioning during psychoanalytic psychotherapies. Journal of the American Psychoanalytic Association, 63(3), 481-509.

Jackson, M. (2002). Weathering the Storms: Psychotherapy for Psychosis. London, Karnac.

Januzzi, G., Verga, M., Piasentin, V., Azzone, P., Vignano, D., \& Freni, S. (1997). The psychodynamic impact of psychopharmacological treatment: Change in defense organization. New Trends in Experimental and Clinical Psychiatry, 13(4), 267-270.

Kernberg, O. F. (1975). Borderline conditions and pathological narcissism. New York, NY: Jason Aronson.

Kernberg, O. F. (1984). Severe personality disorders: Psychotherapeutic strategies. New Haven, CT: Yale University Press.

Kernberg O.F. (2016). What is personality? Journal of Personality Disorders, 30, 145-156.

Klein, M. (1946). Some notes on schizoid mechanisms. International Journal of Psychoanalysis, 27, 99-110.

Kramer, U., de Roten, Y., Perry, J. C., \& Despland, J. N. (2009). Specificities of defense mechanisms in bipolar affective dis- 
order relations with symptoms and therapeutic alliance. Journal of Nervous and Mental Disease, 197, 675-681.

Kramer, U., Despland, J. N., Michel, L., Drapeau, M., \& de Roten, Y. (2010). Change in defense mechanisms and coping over the course of short-term dynamic psychotherapy for adjustment disorder. Journal of Clinical Psychology, 66, 12321241.

Leanza, L., Egloff, L., Studerus, E., Andreou, C., Heitz, U., Ittig, S., Beck, K., Uttinger, M., \& Riecher-Rössler, A. (2018). The relationship between negative symptoms and cognitive functioning in patients at clinical high risk for psychosis. Psychiatry Research, 268, 21-27.

Levy, K. N., Meehan, K. B., Kelly, K. M., Reynoso, J. S., Weber, M., Clarkin, J. F., \& Kernberg, O. F. (2006). Change in attachment patterns and reflective function in a randomized control trial of transference-focused psychotherapy for borderline personality disorder. Journal of Consulting and Clinical Psychology, 74(6), 1027-1040.

Lingiardi, V., \& Boldrini, T. (2019). The diagnostic dilemma of psychosis: Reviewing the historical case of pseudoneurotic schizophrenia. Journal of Nervous and Mental Disease, 207(7), 577-584.

Lieberman, J. A., Small, S. A., \& Girgis, R. R. (2019). Early Detection and Preventive Intervention in Schizophrenia: From Fantasy to Reality. American Journal of Psychiatry, 176(10), 794-810.

Lingiardi, V., \& McWilliams, N. (2017). Psychodynamic diagnostic manual-2nd ed. (PDM-2). New York, NY: Guilford Press.

Maffei, C., Fossati, A., Lingiardi, V., Madeddu, F., Borellini, C., \& Petrachi, M. (1995). Personality maladjustment, defenses, and psychopathological symptoms in nonclinical subjects. Journal of Personality Disorders, 9(4), 330-345.

McGlashan, T. H., Walsh, B. C., Woods, S. W., Addington, J., Cadenhead, K., Cannon, T., ... Walker, E. (2013). Structured interview for psychosis-risk syndromes. New Haven, CT: PRIME Research Clinic, Yale School of Medicine.

Meyer, E.C., Carrion, R.E., Cornblatt, B.A., Addington, J., Cadenhead, K.S., Cannon, T.D., McGlashan, T.H., Perkins, D.O., Tsuang, M.T., Walker, E.F., Woods, S.W., Heinssen, R., Seidman, L.J., group, N. (2014). The relationship of neurocognition and negative symptoms to social and role functioning over time in individuals at clinical high risk in the first phase of the North American Prodrome Longitudinal Study. Schizophrenia Bullettin, 40, 1452-1461.

Millman, Z. B., Gold, J. M., Mittal, V. A., \& Schiffman, J. (2019). The Critical Need for Help-Seeking Controls in Clinical High-Risk Research. Clinical Psychological Science, 7(6), 1171-1189.

Nazzaro, M. P., Boldrini, T., Tanzilli, A., Muzi, L., Giovanardi, G., \& Lingiardi, V. (2017). Does reflective functioning mediate the relationship between attachment and personality? Psychiatry Research, 256, 169-175.

Nelson, B., Lavoie, S., Gaweda, L., Li, E., Sass, L. A., Koren, D., McGorry, P. D., Jack, B. N., Parnas, J., Polari, A., Allott, K., Hartmann, J. A., \& Whitford, T. J. (2019). Testing a neurophenomenological model of basic self disturbance in early psychosis. World Psychiatry, 18(1), 104-105.

Nelson, B., Lavoie, S., Gaweda, L., Li, E., Sass, L. A., Koren, D., McGorry, P. D., Jack, B. N., Parnas, J., Polari, A., Allott, K., Hartmann, J. A., \& Whitford, T. J. (2020). The neurophenomenology of early psychosis: An integrative empirical study. Consciousness and Cognition, 77, 1-17.
Palmier-Claus, J. E., Dunn, G., \& Lewis, S. W. (2012). Emotional and symptomatic reactivity to stress in individuals at ultra-high risk of developing psychosis. Psychological Medicine, 42(5), 1003-1012.

Perry, J. C. (1990). Defense Mechanism Rating Scales (DMRS) (5th ed.). Cambridge: Author.

Parnas, J. (2012). The core Gestalt of schizophrenia. World Psychiatry (WPA), 11(2), 67-69.

Parnas, J., Møller, P., Kircher, T., Thalbitzer, J., Jansson, L., Handest, P., \& Zahavi, D. (2005). EASE: Examination of Anomalous Self-Experience. Psychopathology, 38(5), 236-258.

Perry, J. C., \& Bond, M. (2012). Change in defense mechanisms during long-term dynamic psychotherapy and five-year outcome. American Journal of Psychiatry, 169, 916-925.

Perry, J. C., \& Henry, M. (2004). Studying defense mechanisms in psychotherapy using the Defense Mechanism Rating Scales. In U. Hentschel, G. Smith, J. G. Draguns, \& W. Ehlers (Eds.), Defense mechanisms: Theoretical, research and clinical perspectives (pp. 165-192). Amsterdam: Elsevier.

Perry, J.C., Knoll, M., Tran, V. (2019). Motives, defenses, and conflicts in the dynamic formulation for psychodynamic psychotherapy using the Idiographic Conflict Formulation method. In U Kramer (Ed.), Case Formulation for Personality Disorders. New York: Elsevier, 203-224.

Perry, J.C., Presniak, M.D., Olson, T.R. (2013). Defense Mechanisms in Schizotypal, Borderline, Antisocial, and Narcissistic Personality Disorders, Psychiatry, 76, 32-52.

Presniak, M.D., Olson, T., \& MacGregor, M.W. (2010). The role of defense mechanisms in Borderline and Antisocial personalities. Journal of Personality Assessment, 92, 137-145.

Prout, T.A., Malone, A., Rice, T.R., \& Hoffman, L. (2019). Resilience, defense mechanisms, and implicit emotion regulation in psychodynamic child psychotherapy. Journal of Contemporary Psychotherapy, 49, 235-244.

Sass, L. A., \& Parnas, J. (2003). Schizophrenia, consciousness, and the self. Schizophrenia Bulletin, 29(3), 427-444.

Selzer, M. A., Sullivan, B. T., Carsky, M., \& Terkelsen, K. G. (1989). Working with the person with schizophrenia: The treatment alliance. New York, NY: New York University Press.

Semrad, E. (1967). The organization of ego defenses and object loss. In D. M. Moriarity (Ed.), The loss of loved ones (pp 126-134). Springfield, IL: Charles C. Thomas.

Shaffer, D., Gould, M. S., Brasic, J., Ambrosini, P., Fisher, P., Bird, H., \& Aluwahlia, S. (1983). A Children's Global Assessment Scale (CGAS). Archives of General Psychiatry, 40(11), 1228-1231.

Vaillant, G. E. (1971), Theoretical hierarchy of adaptive ego mechanisms: A 30-year follow-up of 30 men selected for psychological health. Archives of General Psychiatry, 24, 107-118.

Vaillant, G. E. (1992). Ego mechanisms of defense: A guide for clinicians and researchers. Washington: American Psychiatric Press.

Vaillant, G. E. (2011). Involuntary coping mechanisms: A psychodynamic perspective. Dialogues in Clinical Neuroscience, 13, 366-370.

van der Steen, Y., Gimpel-Drees, J., Lataster, T., Viechtbauer, W., Simons, C.J.P., Lardinois, M., ... Myin-Germeys, I. (2017). Clinical high risk for psychosis: the association between momentary stress, affective and psychotic symptoms. Acta Psychiatrica Scandinavica, 136 (1), 63-73. 
Whitty, M. T. (2003). Coping and defending: Age differences in maturity of defence mechanisms and coping strategies. Aging and Mental Health, 7, 123-132.

Winston, B., Winston, A., Samstag, L. W., \& Mu- ran, J. C. (1994). Patient defense/therapist inter- ventions. Psychotherapy, 31, 478-491.

Yung, A. R., \& McGorry, P. D. (1996) The prodromal phase of first-episode psychosis: Past and current conceptualizations. Schizophrenia Bulletin, 22(2), 353-370.
Yung, A. R., McGorry, P. D., McFarlane, C. A., Jackson, H. J., Patton, G. C., \& Rakkar, A. (1996). Monitoring and care of young people at incipient risk of psychosis. Schizophrenia Bulletin, 22(2), 283-303.

Zahavi D. (2005). Subjectivity and Selfhood: Investigating the First-Person Perspective. Cambridge, MA: MIT Press.

Ziegler, D. (2016). Defense mechanisms in Rational Emotive Cognitive Behavior Therapy Personality Theory. Journal of Rational-Emotive \& Cognitive-Behavior Therapy, 34, 135-148. 